\title{
A FINITE ELEMENT METHOD FOR CRACK GROWTH WITHOUT REMESHING
}

\author{
NICOLAS MOËS ${ }^{\dagger}$, JOHN DOLBOW ${ }^{\ddagger}$ AND TED BELYTSCHKO*,§ \\ Department of Mechanical Engineering, Northwestern University, 2145 Sheridan Road, \\ Evanston, IL 60208-3111, U.S.A.
}

\begin{abstract}
SUMMARY
An improvement of a new technique for modelling cracks in the finite element framework is presented. A standard displacement-based approximation is enriched near a crack by incorporating both discontinuous fields and the near tip asymptotic fields through a partition of unity method. A methodology that constructs the enriched approximation from the interaction of the crack geometry with the mesh is developed. This technique allows the entire crack to be represented independently of the mesh, and so remeshing is not necessary to model crack growth. Numerical experiments are provided to demonstrate the utility and robustness of the proposed technique.
\end{abstract}

KEY WORDS: finite elements; fracture

\section{INTRODUCTION}

The modelling of moving discontinuities with the finite element method is cumbersome due to the need to update the mesh to match the geometry of the discontinuity. Several new finite element techniques have been developed to model cracks and crack growth without remeshing. These include the incorporation of a discontinuous mode on an element level [1], a moving mesh technique [2], and an enrichment technique for finite elements based on a partition-of-unity which involves minimal remeshing [3].

In Belytschko and Black [3], curved cracks were treated by mapping the straight crack enriched field. This is not readily applicable to long cracks or three dimensions. In this paper we improve the method by incorporating a discontinuous field across the crack faces away from the crack tip. The method incorporates both the discontinuous Haar function and the near-tip asymptotic functions through a partition of unity method. A crack is grown by redefining the tip location and

\footnotetext{
* Correspondence to: Ted Belytschko, Department of Mechanical Engineering, Northwestern University, 2145 Sheridan Road, Evanston, IL 60208-3111, U.S.A. E-mail: t-belytschko@nwu.edu

$\dagger$ Research Associate

$\ddagger$ DOE Computational Science Graduate Fellow

$\S$ Walter P. Murphy Professor of Mechanical Engineering

Contract/grant sponsor: Office of Naval Research

Contract/grant sponsor: Army Research Office

Contract/grant sponsor: DOE Computational Science Graduate Fellowship Program
} 
and by adding new crack segments. The addition of a discontinuous field allows for the entire crack geometry to be modelled independently of the mesh, and completely avoids the need to remesh as the crack grows.

The present technique exploits the partition of unity property of finite elements identified by Melenk and Babuška [4], which allows local enrichment functions to be easily incorporated into a finite element approximation. A standard approximation is thus 'enriched' in a region of interest by the local functions in conjunction with additional degrees of freedom. For the purpose of fracture analysis, the enrichment functions are the near-tip asymptotic fields and a discontinuous function to represent the jump in displacement across the crack line.

The method differs from the work of Oliver [5], who introduces step functions into the displacement field and then treats the effects on an element level by a multifield approach with an assumed strain field. In the method described in this paper, the displacement field is actually global, but the support of the enrichment functions are local because they are multiplied by nodal shape functions.

This paper is organized as follows. In the next section we present the strong and weak form for linear elastic fracture mechanics. The discrete equations are given in Section 3, which also describes the incorporation of enrichment functions to model cracks. Several numerical examples are given in Section 4. Finally, Section 5 provides a summary and some concluding remarks.

\section{PROBLEM FORMULATION}

In this section, we briefly review the governing equations for elasto-statics and give the associated weak form. Specifically, we consider the case when an internal line is present across which the displacement field may be discontinuous.

\subsection{Governing equations}

Consider the domain $\Omega$ bounded by $\Gamma$. The boundary $\Gamma$ is composed of the sets $\Gamma_{u}, \Gamma_{t}$, and $\Gamma_{c}$, such that $\Gamma=\Gamma_{u} \cup \Gamma_{t} \cup \Gamma_{c}$ as shown in Figure 1. Prescribed displacements are imposed on $\Gamma_{u}$, while tractions are imposed on $\Gamma_{t}$. The crack surface $\Gamma_{c}$ (lines in 2-D and surfaces in 3-D) is assumed to be traction-free.

The equilibrium equations and boundary conditions are

$$
\begin{aligned}
& \boldsymbol{\nabla} \cdot \boldsymbol{\sigma}+\mathbf{b}=0 \quad \text { in } \Omega \\
& \boldsymbol{\sigma} \cdot \mathbf{n}=\overline{\mathbf{t}} \quad \text { on } \Gamma_{t} \\
& \boldsymbol{\sigma} \cdot \mathbf{n}=0 \quad \text { on } \Gamma_{c^{+}} \\
& \boldsymbol{\sigma} \cdot \mathbf{n}=0 \quad \text { on } \Gamma_{c^{-}}
\end{aligned}
$$

where $\mathbf{n}$ is the unit outward normal. In the above, $\boldsymbol{\sigma}$ is the Cauchy stress, and $\mathbf{b}$ is the body force per unit volume.

In the present investigation, we consider small strains and displacements. The kinematics equations therefore consist of the strain-displacement relation

$$
\varepsilon=\varepsilon(\mathbf{u})=\nabla_{\mathrm{s}} \mathbf{u}
$$

where $\nabla_{\mathrm{s}}$ is the symmetric part of the gradient operator, and the boundary conditions

$$
\mathbf{u}=\overline{\mathbf{u}} \quad \text { on } \Gamma_{u}
$$




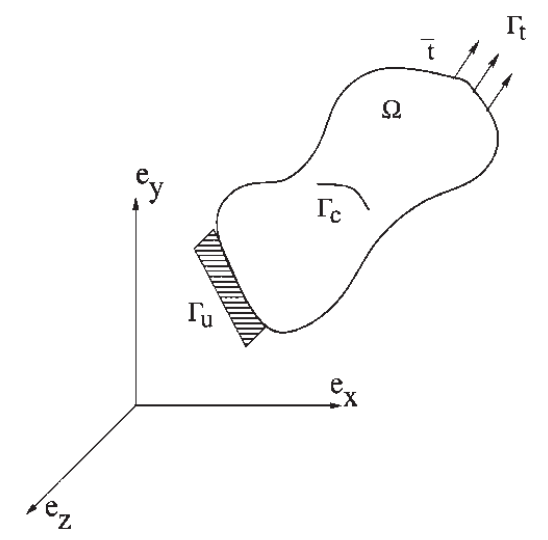

Figure 1. Body with internal boundary subjected to loads

The constitutive relation is given by Hooke's law:

$$
\boldsymbol{\sigma}=\mathbf{C}: \boldsymbol{\varepsilon}
$$

where $\mathbf{C}$ is the Hooke tensor.

\subsection{Weak form}

The space of admissible displacement fields is defined by

$$
\mathscr{U}=\left\{\mathbf{v} \in \mathscr{V}: \mathbf{v}=\overline{\mathbf{u}} \text { on } \Gamma_{u} \mathbf{v} \text { discontinuous on } \Gamma_{c}\right\}
$$

where the space $\mathscr{V}$ is related to the regularity of the solution. The details on this matter when the domain contains an internal boundary or re-entrant corner may be found in Babuška and Rosenzweig [6] and Grisvard [7]. We note that the space $\mathscr{V}$ allows for discontinuous functions across the crack line. The test function space is defined similiarly as

$$
\mathscr{U}_{0}=\left\{\mathbf{v} \in \mathscr{V}: \mathbf{v}=0 \text { on } \Gamma_{u} \mathbf{v} \text { discontinuous on } \Gamma_{c}\right\}
$$

The weak form of the equilibrium equations is given by

$$
\int_{\Omega} \boldsymbol{\sigma}: \boldsymbol{\varepsilon}(\mathbf{v}) \mathrm{d} \Omega=\int_{\Omega} \mathbf{b} \cdot \mathbf{v} \mathrm{d} \Omega+\int_{\Gamma_{t}} \overline{\mathbf{t}} \cdot \mathbf{v} \mathrm{d} \Gamma \quad \forall \mathbf{v} \in \mathscr{U}_{0}
$$

Using the constitutive relation and the kinematics constraints in the weak form, the problem is to find $\mathbf{u} \in \mathscr{U}$ such that

$$
\int_{\Omega} \boldsymbol{\varepsilon}(\mathbf{u}): \mathbf{C}: \boldsymbol{\varepsilon}(\mathbf{v}) \mathrm{d} \Omega=\int_{\Omega} \mathbf{b} \cdot \mathbf{v} \mathrm{d} \Omega+\int_{\Gamma_{t}} \overline{\mathbf{t}} \cdot \mathbf{v} \mathrm{d} \Gamma \quad \forall \mathbf{v} \in \mathscr{U}_{0}
$$

It is shown in Belytschko and Black [3] that the above is equivalent to the strong form (1), including the traction-free conditions on the two crack faces. In contrast to boundary element techniques, this enables the method to be easily extended to non-linear problems. 


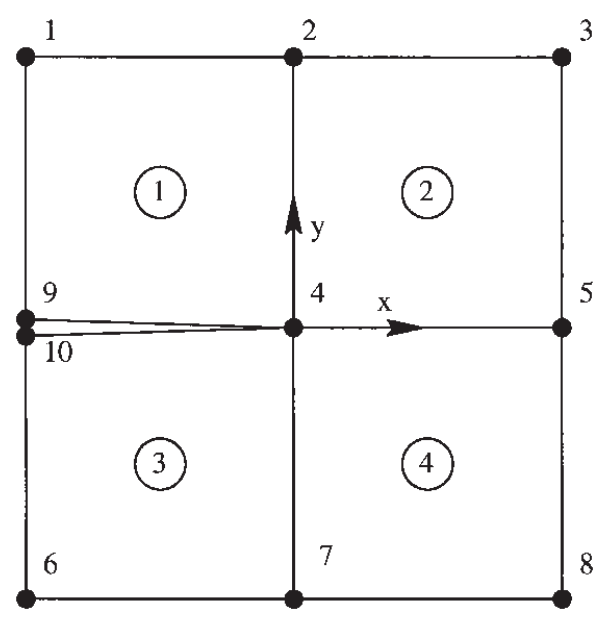

Figure 2. Finite element mesh near a crack tip, the circled numbers are element numbers

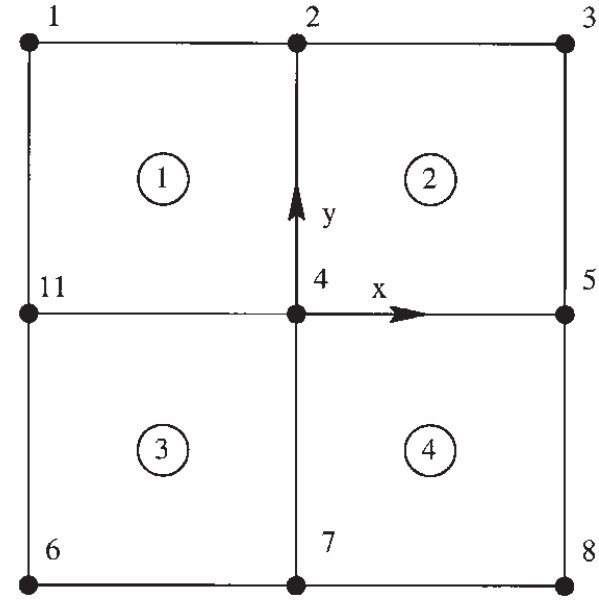

Figure 3. Regular mesh without a crack

\section{DISCRETIZATION}

In this section, we present the construction of approximations which are discontinuous across a given line or surface and include the asymptotic near-tip fields. After examining a simple case, criteria for selecting the enriched nodes for an arbitrary mesh and crack geometry are given. We also discuss the modifications necessary to accurately integrate the weak form, and review the numerical procedure to calculate the stress intensity factors.

\subsection{Crack modelling using discontinuous enrichment}

The model consists of a standard finite element model and a crack representation which is independent of the elements. In order to introduce the notion of discontinuous enrichment, we first consider a simple case of an edge crack modelled by four elements as shown in Figure 2. For convenience, the local co-ordinate system is aligned with the crack tip. We wish to illustrate how an equivalent discrete space can be constructed with the mesh shown in Figure 3 and the addition of a discontinuous field.

The finite element approximation associated with the mesh in Figure 2 is

$$
\mathbf{u}^{h}=\sum_{i=1}^{10} \mathbf{u}_{i} \phi_{i}
$$

where $\mathbf{u}_{i}$ is the displacement at node $i$ and $\phi_{i}$ is the bilinear shape function associated with node $i$. Each shape function $\phi_{i}$ has a compact support $\omega_{i}$ given by the union of the elements connected to node $i$.

Defining $\mathbf{a}$ and $\mathbf{b}$ as

$$
\mathbf{a}=\frac{\mathbf{u}_{9}+\mathbf{u}_{10}}{2}, \quad \mathbf{b}=\frac{\mathbf{u}_{9}-\mathbf{u}_{10}}{2}
$$

We can express $\mathbf{u}_{9}$ and $\mathbf{u}_{10}$ in terms of $\mathbf{a}$ and $\mathbf{b}$

$$
\mathbf{u}_{9}=\mathbf{a}+\mathbf{b}, \quad \mathbf{u}_{10}=\mathbf{a}-\mathbf{b}
$$




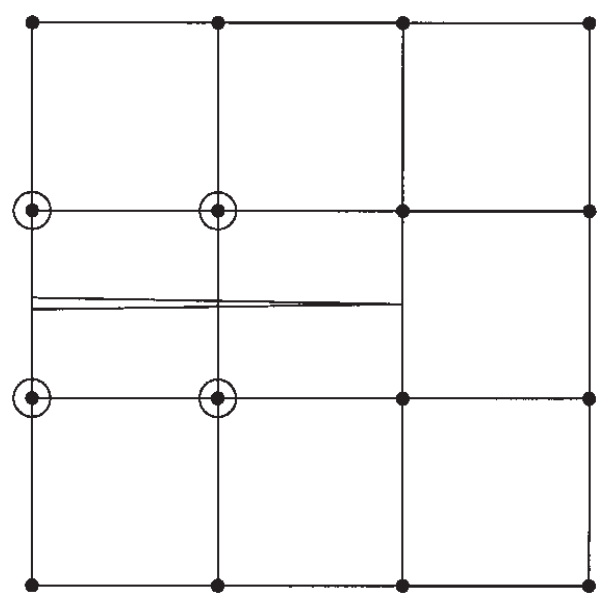

Figure 4. Crack not aligned with a mesh, the circled nodes are enriched with the discontinuous function

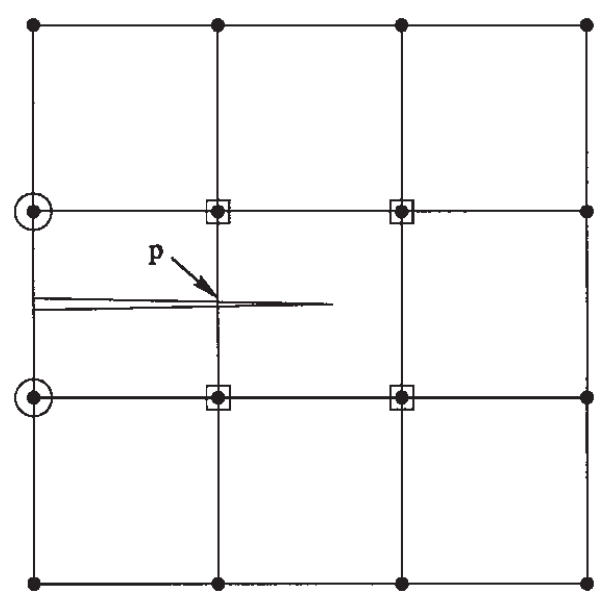

Figure 5. Crack not aligned with a mesh, the circled nodes are enriched with the discontinuous function and the squared nodes with the tip enrichment functions. Enrichment with only the discontinuous function shortens the crack to point $p$

Then replacing $\mathbf{u}_{9}$ and $\mathbf{u}_{10}$ in terms of $\mathbf{a}$ and $\mathbf{b}$ in (9) yields

$$
\mathbf{u}^{h}=\sum_{i=1}^{8} \mathbf{u}_{i} \phi_{i}+\mathbf{a}\left(\phi_{9}+\phi_{10}\right)+\mathbf{b}\left(\phi_{9}+\phi_{10}\right) H(\mathbf{x})
$$

where $H(\mathbf{x})$ is referred to here as a discontinuous, or 'jump' function. This is defined in the local crack co-ordinate system as

$$
H(x, y)=\left\{\begin{aligned}
1 & \text { for } y>0 \\
-1 & \text { for } y<0
\end{aligned}\right.
$$

such that $H(\mathbf{x})=1$ on element 1 and -1 on element 3 , respectively.

If we now consider the mesh in Figure $3, \phi_{9}+\phi_{10}$ can be replaced by $\phi_{11}$, and $\mathbf{a}$ by $\mathbf{u}_{11}$. The finite element approximation now reads

$$
\mathbf{u}^{h}=\sum_{i=1}^{8} \mathbf{u}_{i} \phi_{i}+\mathbf{u}_{11} \phi_{11}+\mathbf{b} \phi_{11} H(\mathbf{x})
$$

The first two terms on the right-hand side represent the classical finite element approximation, whereas the last one represents the addition of a discontinuous enrichment. In other words, when a crack is modeled by a mesh as in Figure 2, we may interpret the finite element space as the sum of one which does not model the crack (such as Figure 3 ) and a discontinuous enrichment.

The previous derivation provides insight into the extension of the technique for the case when the crack does not align with the mesh. The key issues are the selection of the appropriate nodes to enrich, and the form of the associated enrichment functions. In terms of enrichment with the jump function, we adopt the convention that a node is enriched if its support is cut by the crack into two disjoint pieces. This rule is seen to be consistent with the previous example, in which only node 11 was enriched. Figure 4 illustrates the application of this rule when the crack is not aligned with the element edges, in which the circled nodes are enriched with the jump function. 


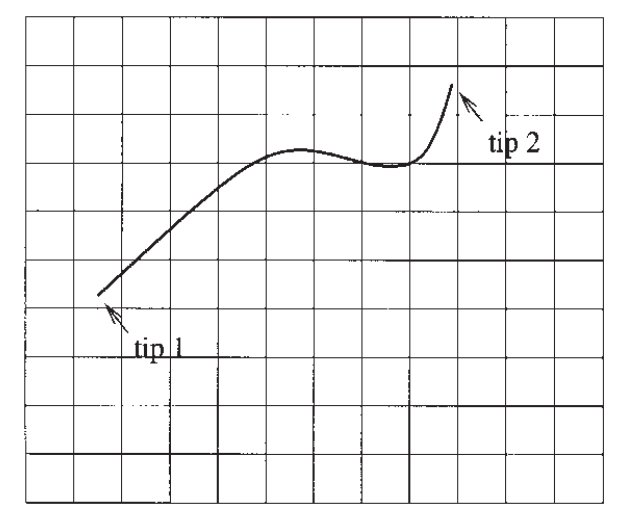

Figure 6. An arbitrary crack placed on a mesh

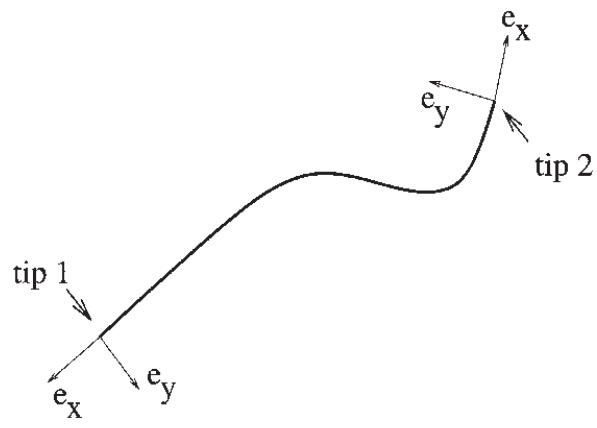

Figure 7. Local axes for the polar co-ordinates at the two crack tips

In a more general case such as that shown in Figure 5, the crack tip will not coincide with an element edge, and in this instance the discontinuity cannot be adequately described using only a function such as $H(\mathbf{x})$. The jump enrichment of the circled nodes in this case only provides for the modelling of the discontinuity up until point $p$. To seamlessly model the entire discontinuity along the crack, the squared nodes are enriched with the asymptotic crack tip functions with the technique developed in Belytschko and Black [3]. For example, for the discretization shown in Figure 5, the approximation takes the form

$$
\mathbf{u}^{h}=\sum_{i \in I} \mathbf{u}_{i} \phi_{i}+\sum_{j \in J} \mathbf{b}_{j} \phi_{j} H(\mathbf{x})+\sum_{k \in K} \phi_{k}\left(\sum_{l=1}^{4} \mathbf{c}_{k}^{l} F_{l}(\mathbf{x})\right)
$$

in which $J$ is the set of circled nodes and $K$ the set of squared nodes. The functions $F_{l}(\mathbf{x})$ are defined as

$$
\left\{F_{l}(r, \theta)\right\} \equiv\left\{\sqrt{r} \sin \left(\frac{\theta}{2}\right), \sqrt{r} \cos \left(\frac{\theta}{2}\right), \sqrt{r} \sin \left(\frac{\theta}{2}\right) \sin (\theta), \sqrt{r} \cos \left(\frac{\theta}{2}\right) \sin (\theta)\right\}
$$

where $(r, \theta)$ are the local polar co-ordinates at the crack tip. Note that the first function in (16), $\sqrt{r} \sin (\theta / 2)$, is discontinuous across the crack faces whereas the last three functions are continuous. The function $H(\mathbf{x})$ is given by (13) where the local axes are taken to be aligned with the crack tip as in Figure 2.

We now generalize to the case of an arbitrary crack, as shown in Figure 6. The approximation takes the form:

$$
\mathbf{u}^{h}=\sum_{i \in I} \mathbf{u}_{i} \phi_{i}+\sum_{j \in J} \mathbf{b}_{j} \phi_{j} H(\mathbf{x})+\sum_{k \in K_{1}} \phi_{k}\left(\sum_{l=1}^{4} \mathbf{c}_{k}^{l 1} F_{l}^{1}(\mathbf{x})\right)+\sum_{k \in K_{2}} \phi_{k}\left(\sum_{l=1}^{4} \mathbf{c}_{k}^{l 2} F_{l}^{2}(\mathbf{x})\right)
$$

where $K_{1}$ and $K_{2}$ are the sets of nodes to be enriched for the first and second crack tip, respectively. The precise definition of these two sets as well as the set $J$ will be given further. The functions $F_{l}^{1}(\mathbf{x})$ and $F_{l}^{2}(\mathbf{x})$ are identical to the ones given in (16), with $\left(r_{1}, \theta_{1}\right)$ and $\left(r_{2}, \theta_{2}\right)$ being defined in the local crack tip system at tips 1 and 2, respectively as shown in Figure 7.

The jump function $H(\mathbf{x})$ is defined as follows. The crack is considered to be a curve parametrized by the curvilinear co-ordinate $s$, as in Figure 8. The origin of the curve is taken to coincide with one of the crack tips. Given a point $\mathbf{x}$ in the domain, we denote by $\mathbf{x}^{*}$ the closest point on the 


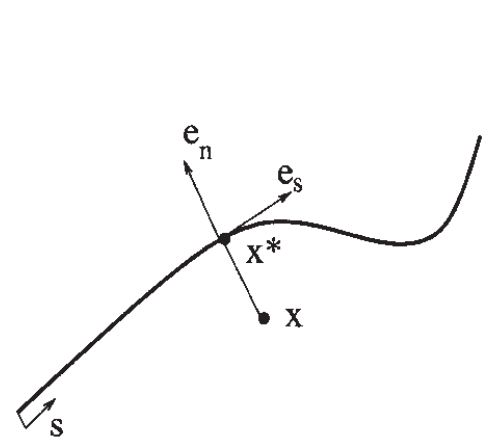

a)

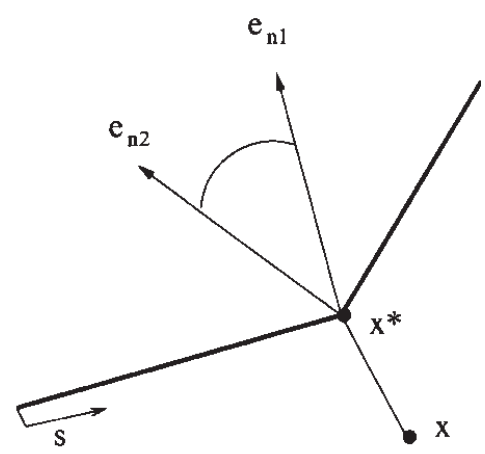

b)

Figure 8. Illustration of normal and tangential co-ordinates for a smooth crack: (a) and for a crack with a kink; (b) $\mathbf{x}^{*}$ is the closest point to $\mathbf{x}$ on the crack. In both of the above cases, the jump function $H(\mathbf{x})=-1$

crack to $\mathbf{x}$. At $\mathbf{x}^{*}$, we construct the tangential and normal vector to the curve, $\mathbf{e}_{s}$ and $\mathbf{e}_{n}$, with the orientation of $\mathbf{e}_{n}$ taken such that $\mathbf{e}_{s} \wedge \mathbf{e}_{n}=\mathbf{e}_{z}$. The sign of the step function $H(\mathbf{x})$ is then given by the sign of the scalar product $\left(\mathbf{x}-\mathbf{x}^{*}\right) \cdot \mathbf{e}_{n}$. In the case of a kinked crack as shown in Figure $8(\mathrm{~b})$, where no unique normal but a cone of normals is defined at $\mathbf{x}^{*}, H(\mathbf{x})=1$ if the vector $\left(\mathbf{x}-\mathbf{x}^{*}\right)$ belongs to the cone of normals at $\mathbf{x}^{*}$ and -1 otherwise.

We now turn to the definitions of $J, K_{1}$ and $K_{2}$. We shall denote by $\mathbf{x}_{1}$ and $\mathbf{x}_{2}$ the location of the crack tips 1 and 2, respectively, and by $C$ the geometry of the crack. The sets $K_{1}$ and $K_{2}$ consist of those nodes for which the closure of the nodal support contains crack tip 1 or 2 , respectively. The set $J$ is the set of nodes whose support is intersected by the crack and do not belong to $K_{1}$ or $K_{2}$.

$$
\begin{aligned}
K_{1} & =\left\{k \in I: \mathbf{x}_{1} \in \bar{\omega}_{k}\right\} \\
K_{2} & =\left\{k \in I: \mathbf{x}_{2} \in \bar{\omega}_{k}\right\} \\
J & =\left\{j \in I: \omega_{j} \cap C \neq \emptyset, j \notin K_{1}, j \notin K_{2}\right\}
\end{aligned}
$$

Remarks.

(1) From the definition of the sets $K_{1}, K_{2}$, and $J$, we see that any node whose support is intersected by the crack will be enriched by a discontinuous function: of $H$ type for the nodes in $J$ and of $F$ type for the nodes in $K_{1}$ and $K_{2}$. So, the displacement is allowed to be discontinous along the full extent of the crack.

(2) The treatment of a free edge crack is similar, with either set $K_{1}$ or $K_{2}$ being empty.

(3) The set $K_{1}$ and/or $K_{2}$ can be enlarged to include all nodes within a characteristic radius of the associated crack tip, in which region the asymptotic near-tip fields are assumed to dominate the solution.

(4) The case of multiple cracks is obtained by considering the proper sets $J, K_{1}$ and $K_{2}$ for each crack.

(5) For the case when multiple crack segments are enriched with the near-tip fields, a mapping is used to align the discontinuity with the crack geometry [3]. 

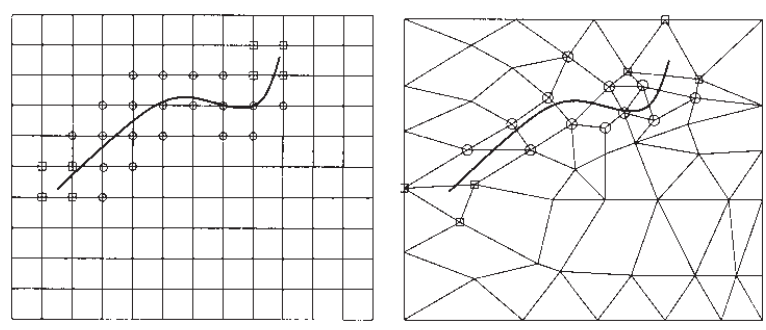

Figure 9. Crack on a uniform mesh (left) and on a non-uniform mesh (right). The circled nodes are enriched by the jump function whereas the squared nodes are enriched by the crack tip functions

Figure 9 illustrates the sets $J$ (circled nodes), $K_{1}$ (squared nodes near tip 1) and $K_{2}$ (squared nodes near tip 2) for a uniform and non uniform mesh.

\subsection{Extension to higher-order finite elements}

In the previous section, we have shown how a discontinuity is introduced into a finite element approximation with a local enrichment. We considered that the finite element approximation prior to the introduction of the discontinuity was of the first order, i.e. one with linear displacements for triangular elements and bilinear for quadrilaterals. The extension to higher-order finite element approximations is straightforward.

Consider hierarchical based $p$-order finite elements [8]: a first-order finite element approximation uses the classical nodal shape functions whereas a higher-order approximation introduces additional edge and element (bubble) shape functions. The enrichment strategy must therefore consider these additional edge and internal degrees of freedom.

Let $D$ be the set of all the degrees of freedom for a given mesh and order of approximation. Each degree of freedom $\mathbf{u}_{i}, i \in D$, is a vector with a $x$ and $y$ displacement components. The finite element approximation, prior to the introduction of the discontinuity, is given by

$$
\mathbf{u}^{h}=\sum_{i \in D} \mathbf{u}_{i} \phi_{i}
$$

where $\phi_{i}$ is the shape function associated with the $i$ th degree of freedom.

In order to select the degrees of freedom to be enriched by the Haar function, we extend the rule introduced in the previous section: 'a node is enriched if its support is cut by the crack' to 'a degree of freedom is enriched if it's support is cut by the crack'. The support of an edge degree of freedom is made of the element(s) connected to it and the support of an element degree of freedom is the element itself. More precisely, the subset $D^{\prime} \subset D$ of the degrees of freedom to be enriched by the Haar function must have their support cut by the crack but must not contain any of the crack tips. Formally, in the case of a crack with two tips:

$$
D^{\prime}=\left\{i \in D: \omega_{i} \cap C \neq \emptyset, \mathbf{x}_{1} \notin \bar{\omega}_{i}, \mathbf{x}_{2} \notin \bar{\omega}_{i}\right\}
$$

The finite element approximation after the introduction of the discontinuity is then

$$
\mathbf{u}^{h}=\sum_{i \in D} \mathbf{u}_{i} \phi_{i}+\sum_{j \in D^{\prime}} \mathbf{b}_{j} \phi_{j} H(\mathbf{x})+\sum_{k \in K_{1}} \phi_{k}\left(\sum_{l=1}^{4} \mathbf{c}_{k}^{l 1} F_{l}^{1}(\mathbf{x})\right)+\sum_{k \in K_{2}} \phi_{k}\left(\sum_{l=1}^{4} \mathbf{c}_{k}^{l 2} F_{l}^{2}(\mathbf{x})\right)
$$




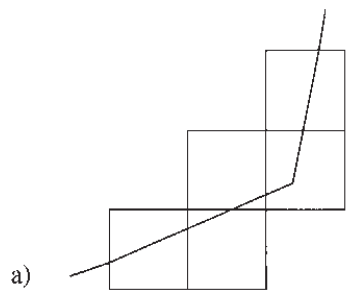

b)
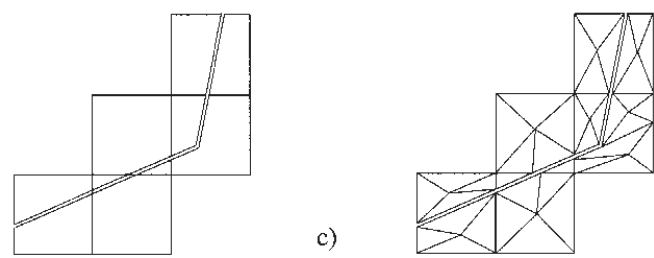

Figure 10. Generation of subpolygons for the quadrature of the weak form in: (a) those elements cut by a crack. The polygons (b) formed from the intersection of the crack and the element geometries are triangulated as in (c) to create the element subdomains

where

$$
K_{1}=\left\{k \in \tilde{D}: \mathbf{x}_{1} \in \bar{\omega}_{k}\right\}, \quad K_{2}=\left\{k \in \tilde{D}: \mathbf{x}_{2} \in \bar{\omega}_{k}\right\}
$$

and $\tilde{D} \subset D$ is the subset of nodal degrees of freedom. We therefore keep a nodal-based enrichment for the near-tip enrichment. From the expression for approximation (23), we notice that the jump displacement across the crack is now described by higher-order functions along the crack faces. In addition, we have not assumed a uniform $p$ order of approximation.

\subsection{Numerical integration of the weak form}

For elements cut by the crack and enriched with the jump function $H(\mathbf{x})$, we make a modification to the element quadrature routines in order to accurately assemble the contribution to the weak form on both sides of the discontinuity. As the crack is allowed to be arbitrarily oriented in an element, the use of standard Gauss quadrature may not adequately integrate the discontinuous field. If the integration of the the jump enrichment is indistinguishable from that of a constant function, spurious singular modes can appear in the system of equations. In this section, we present the modifications made to the numerical integration scheme for elements cut by a crack.

The discrete weak form is normally constructed with a loop over all elements, as the domain is approximated by

$$
\Omega=\sum_{e} \Omega_{e}
$$

where $\Omega_{e}$ is the element subdomain. For elements cut by a crack, we define the element subdomain to be a sum of a set of subpolygons whose boundaries align with the crack geometry

$$
\Omega_{e}=\sum_{s} \Omega_{S}
$$

In two dimensions, the triangles shown in Figure 10 work well. Simpler schemes, such as the trapezoids used by Fish [9] may also perform adequately. It is emphasized that the subpolygons are only necessary for integration purposes; no additional degrees of freedom are associated with their construction. In the integration of the weak form, the element loop is replaced by a loop over the subpolygons for those elements cut by the crack.

\subsection{Crack growth and stress intensity factor evaluation}

In this section, we briefly review the criterion used to specify the direction of crack growth. In addition, we describe the domain form of the interaction integral for the extraction of mixed-mode stress intensity factors. 
Under general mixed-mode loadings, the asymptotic near-tip circumferential and shear stresses take the following form:

$$
\left\{\begin{array}{c}
\sigma_{\theta \theta} \\
\sigma_{r \theta}
\end{array}\right\}=\frac{K_{\mathrm{I}}}{\sqrt{2 \pi r}} \frac{1}{4}\left\{\begin{array}{c}
3 \cos (\theta / 2)+\cos (3 \theta / 2) \\
\sin (\theta / 2)+\sin (3 \theta / 2)
\end{array}\right\}+\frac{K_{\mathrm{II}}}{\sqrt{2 \pi r}} \frac{1}{4}\left\{\begin{array}{c}
-3 \sin (\theta / 2)-3 \sin (3 \theta / 2) \\
\cos (\theta / 2)+3 \cos (3 \theta / 2)
\end{array}\right\}
$$

Among the criteria for determining the growth direction are: (1) the maximum energy release rate criterion [10], (2) the maximum circumferential stress criterion or the maximum principal stress criterion [11] and (3) the minimum strain energy density criterion [12].

In this paper, we use the maximum circumferential stress criterion, which states that the crack will propagate from its tip in a direction $\theta_{c}$ so that the circumferential stress $\sigma_{\theta \theta}$ is maximum. The circumferential stress in the direction of crack propagation is a principal stress. Therefore, the critical angle $\theta_{c}$ defining the radial direction of propagation can be determined by setting the shear stress in (27) to zero. After a few manipulations, the following expression is obtained:

$$
\frac{1}{\sqrt{2 \pi r}} \cos \left(\frac{\theta}{2}\right)\left[\frac{1}{2} K_{\mathrm{I}} \sin (\theta)+\frac{1}{2} K_{\mathrm{II}}(3 \cos (\theta)-1)\right]=0
$$

This leads to the equation defining the angle of crack propagation $\theta_{c}$ in the tip co-ordinate system.

$$
K_{\mathrm{I}} \sin \left(\theta_{c}\right)+K_{\mathrm{II}}\left(3 \cos \left(\theta_{c}\right)-1\right)=0
$$

Solving this equation gives

$$
\theta_{c}=2 \arctan \frac{1}{4}\left(K_{\mathrm{I}} / K_{\mathrm{II}} \pm \sqrt{\left(K_{\mathrm{I}} / K_{\mathrm{II}}\right)^{2}+8}\right)
$$

The stress intensity factors are computed using domain forms of the interaction integrals [13, 14]. For completeness these are discussed here. The coordinates are taken to be the local crack tip co-ordinates with the $x_{1}$-axis parallel to the crack faces. For general mixed-mode problems we have the following relationship between the value of the $J$-integral and the stress intensity factors

$$
J=\frac{K_{\mathrm{I}}^{2}}{E^{*}}+\frac{K_{\mathrm{II}}^{2}}{E^{*}}
$$

where $E^{*}$ is defined in terms of material parameters $E$ (Young's modulus) and $v$ (poisson's ratio) as

$$
E^{*}= \begin{cases}E & \text { plane stress } \\ \frac{E}{1-v^{2}} & \text { plane strain }\end{cases}
$$

Two states of a cracked body are considered. State $1,\left(\sigma_{i j}^{(1)}, \varepsilon_{i j}^{(1)}, u_{i}^{(1)}\right)$, corresponds to the present state and state $2,\left(\sigma_{i j}^{(2)}, \varepsilon_{i j}^{(2)}, u_{i}^{(2)}\right)$, is an auxiliary state which will be chosen as the asymptotic fields for Modes I or II. The $J$-integral for the sum of the two states is

$$
J^{(1+2)}=\int_{\Gamma}\left[\frac{1}{2}\left(\sigma_{i j}^{(1)}+\sigma_{i j}^{(2)}\right)\left(\varepsilon_{i j}^{(1)}+\varepsilon_{i j}^{(2)}\right) \delta_{1 j}-\left(\sigma_{i j}^{(1)}+\sigma_{i j}^{(2)}\right) \frac{\partial\left(u_{i}^{(1)}+u_{i}^{(2)}\right)}{\partial x_{1}}\right] n_{j} \mathrm{~d} \Gamma
$$

Expanding and rearranging terms gives

$$
J^{(1+2)}=J^{(1)}+J^{(2)}+I^{(1,2)}
$$




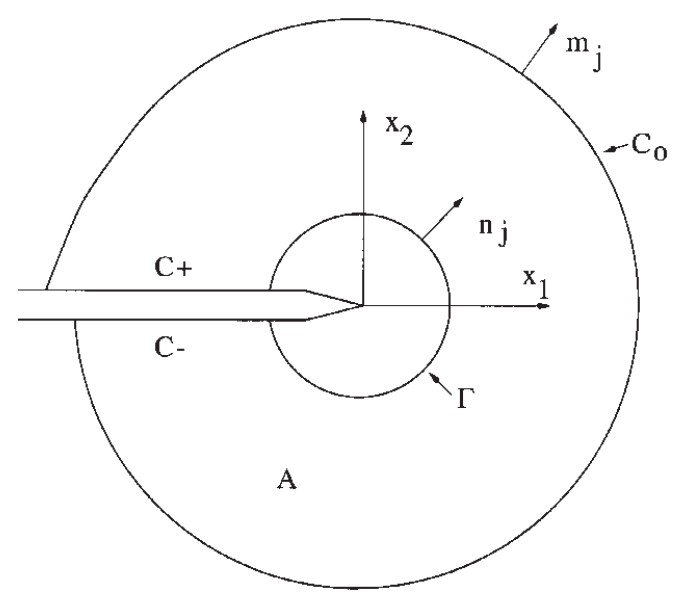

Figure 11. Conventions at crack tip. Domain $\mathrm{A}$ is enclosed by $\Gamma, C_{+}, C_{-}$, and $C_{0}$. Unit normal $m_{j}=n_{j}$ on $C_{+}, C_{-}$, and $C_{0}$ and $m_{j}=-n_{j}$ on $\Gamma$

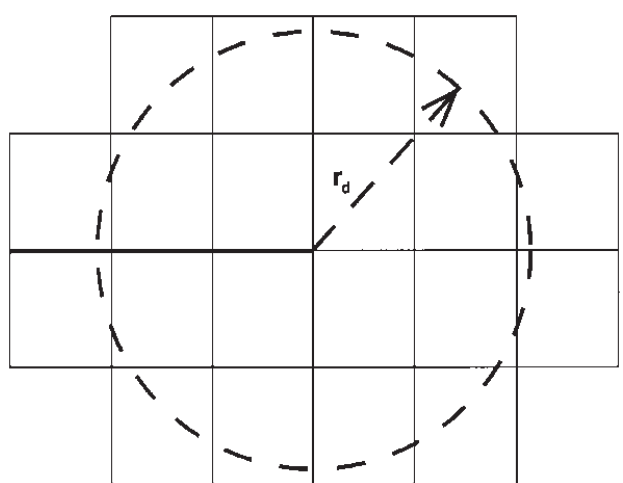

Figure 12. Elements selected about the crack tip for calculation of the interaction integral

where $I^{(1,2)}$ is called the interaction integral for states 1 and 2

$$
I^{(1,2)}=\int_{\Gamma}\left[W^{(1,2)} \delta_{1 j}-\sigma_{i j}^{(1)} \frac{\partial\left(u_{i}^{2}\right)}{\partial x_{1}}-\sigma_{i j}^{(2)} \frac{\partial\left(u_{i}^{1}\right)}{\partial x_{1}}\right] n_{j} \mathrm{~d} \Gamma
$$

where $W^{(1,2)}$ is the interaction strain energy

$$
W^{(1,2)}=\sigma_{i j}^{(1)} \varepsilon_{i j}^{(2)}=\sigma_{i j}^{(2)} \varepsilon_{i j}^{(1)}
$$

Writing equation (31) for the combined states gives after rearranging terms

$$
J^{(1+2)}=J^{(1)}+J^{(2)}+\frac{2}{E^{*}}\left(K_{\mathrm{I}}^{(1)} K_{\mathrm{I}}^{(2)}+K_{\mathrm{II}}^{(1)} K_{\mathrm{II}}^{(2)}\right)
$$

Equating (34) with (37) leads to the following relationship:

$$
I^{(1,2)}=\frac{2}{E^{*}}\left(K_{\mathrm{I}}^{(1)} K_{\mathrm{I}}^{(2)}+K_{\mathrm{II}}^{(1)} K_{\mathrm{II}}^{(2)}\right)
$$

Making the judicious choice of state 2 as the pure Mode I asymptotic fields with $K_{\mathrm{I}}^{(2)}=1$ gives mode I stress intensity factor for state 1 in terms of the interaction integral

$$
K_{\mathrm{I}}^{(1)}=\frac{2}{E^{*}} I^{(1, \text { Mode I })}
$$

Mode II stress intensity factor can be determined in a similiar fashion.

The contour integral (35) is not in a form best suited for finite element calculations. We therefore recast the integral into an equivalent domain form by multiplying the integrand by a sufficiently smooth weighting function $q(\mathbf{x})$ which takes a value of unity on an open set containing the crack tip and vanishes on an outer prescribed contour $C_{0}$. Then for each contour $\Gamma$ as in Figure 11, assuming the crack faces are traction free and straight in the region $A$ bounded by the contour $C_{0}$, the interaction integral may be written as

$$
I^{(1,2)}=\int_{C}\left[W^{(1,2)} \delta_{1 j}-\sigma_{i j}^{(1)} \frac{\partial u_{i}^{2}}{\partial x_{1}}-\sigma_{i j}^{(2)} \frac{\partial u_{i}^{1}}{\partial x_{1}}\right] q m_{j} \mathrm{~d} \Gamma
$$




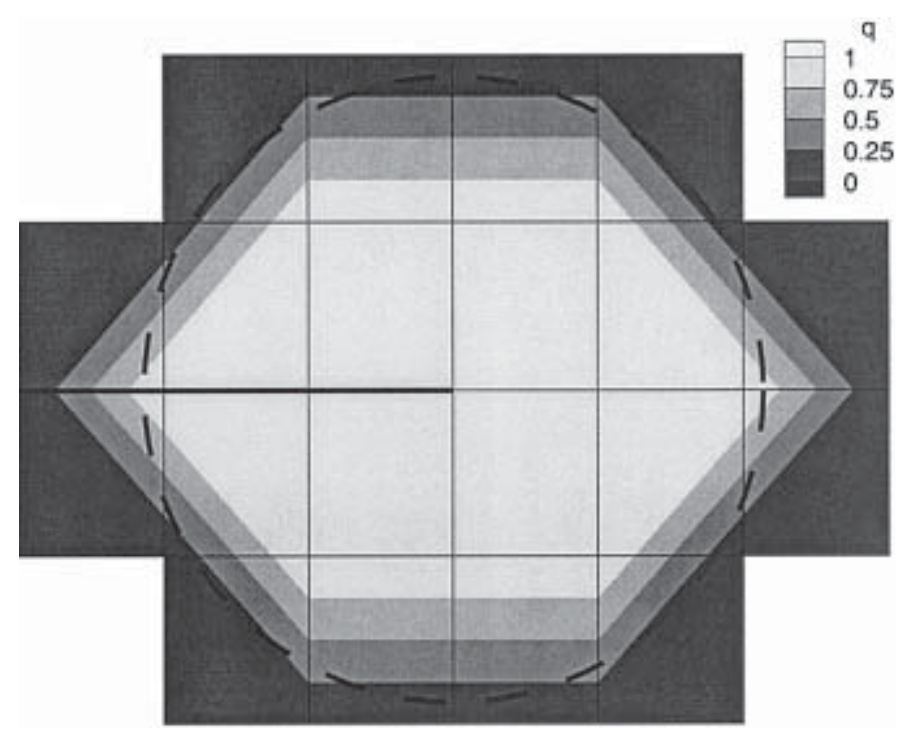

Figure 13. Weight function $q$ on the elements

where the contour $C=\Gamma+C_{+}+C_{-}+C_{0}$ and $\mathbf{m}$ is the unit outward normal to the contour $C$. Now using the divergence theorem and passing to the limit as the contour $\Gamma$ is shrunk to the crack tip, gives the following equation for the interaction integral in domain form:

$$
I^{(1,2)}=\int_{A}\left[\sigma_{i j}^{(1)} \frac{\partial u_{i}^{2}}{\partial x_{1}}+\sigma_{i j}^{(2)} \frac{\partial u_{i}^{1}}{\partial x_{1}}-W^{(1,2)} \delta_{1 j}\right] \frac{\partial q}{\partial x_{j}} \mathrm{~d} A
$$

where we have used the relations $m_{j}=-n_{j}$ on $\Gamma$ and $m_{j}=n_{j}$ on $C_{0}, C_{+}$and $C_{-}$.

For the numerical evaluation of the above integral, the domain $A$ is set from the collection of elements about the crack tip. In this paper, we first determine the characteristic length of an element touched by the crack tip and designate this quantity as $h_{\text {local }}$. For two-dimensional analysis, this quantity is calculated as the square root of the element area. The domain $A$ is then set to be all elements which have a node within a ball of radius $r_{d}$ about the crack tip.

Figure 12 shows a typical set of elements for the domain $A$ with the domain radius $r_{d}$ taken to be twice the length $h_{\text {local }}$. Figure 13 shows the contour plot of the weight function $q$ for these elements. The $q$ function is taken to have a value of unity for all nodes within the ball $r_{d}$, and zero on the outer contour. The function is then easily interpolated within the elements using the nodal shape functions.

\section{NUMERICAL EXAMPLES}

In this section, we present several numerical examples of cracks and crack growth under the assumptions of plane strain two-dimensional elasticity. We begin with a simple example of an edge crack to demonstrate the robustness of the discretization scheme, and then present results for more complicated geometries.

In all of the following examples, the material properties are assumed to be that of glass with young's modulus $100 \mathrm{kpsi}$, and poisson ratio $0 \cdot 3$. The calculation of the stress intensity factors is performed with the domain form of the interaction integral as detailed in the previous section. 


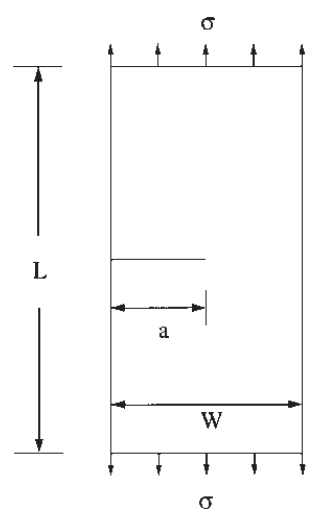

Figure 14. The geometry of the edge crack problem for the robustness and shear studies. The parameters are $a / W=1 / 2$, $L / W=16 / 7, W=7$
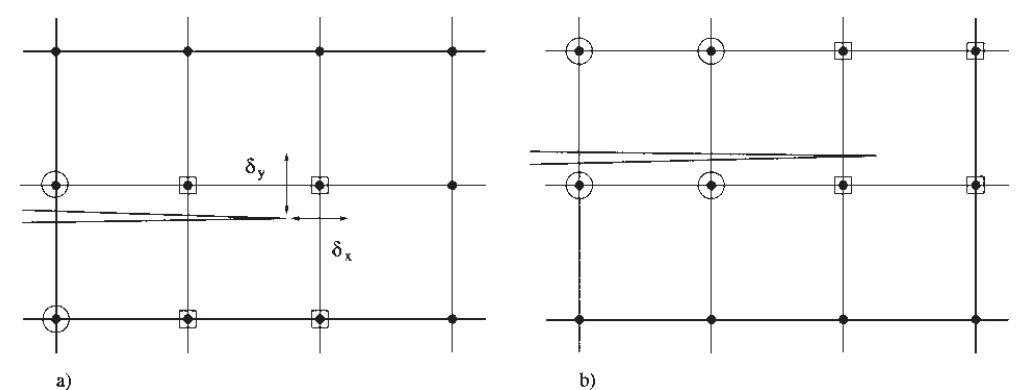

Figure 15. Zoom of the mesh in the vicinity of the crack tip, with: (a) the initial configuration and $\delta_{x}, \delta_{y}$ shown. The enrichment is also shown for; (b) the final configuration. The circled nodes are enriched with the jump function and the squared nodes with the near-tip functions

\subsection{Robustness analysis}

Consider the geometry shown in Figure 14: a plate of width $w$ and height $L$ with an edge crack of length $a$. We analyse the influence of the location of the crack with respect to the mesh on the $K_{\mathrm{I}}$ stress intensity factor when the position of the crack is perturbed by $\delta x$ in the $X$-direction and $\delta y$ in the $Y$-direction. The model is a uniform mesh of $24 \times 48$ 4-noded quadrilateral elements.

In this study, several different discretizations are obtained depending on the position of the crack with respect to the mesh. Two cases are shown in Figure 15. Depending on the location of the crack tip, the total number of degrees of freedom varies from 2501 to 2541 . The purpose of the study is to compare the accuracy of the solution when the crack is aligned with the mesh to the case when it is slightly offset.

The exact solution for this problem is given by [15]

$$
K_{\mathrm{I}}=C \sigma \sqrt{a \pi}
$$

where $C$ is a finite-geometry correction factor:

$$
C=1 \cdot 12-0.231\left(\frac{a}{W}\right)+10 \cdot 55\left(\frac{a}{W}\right)^{2}-21 \cdot 72\left(\frac{a}{W}\right)^{3}+30 \cdot 39\left(\frac{a}{W}\right)^{4}
$$




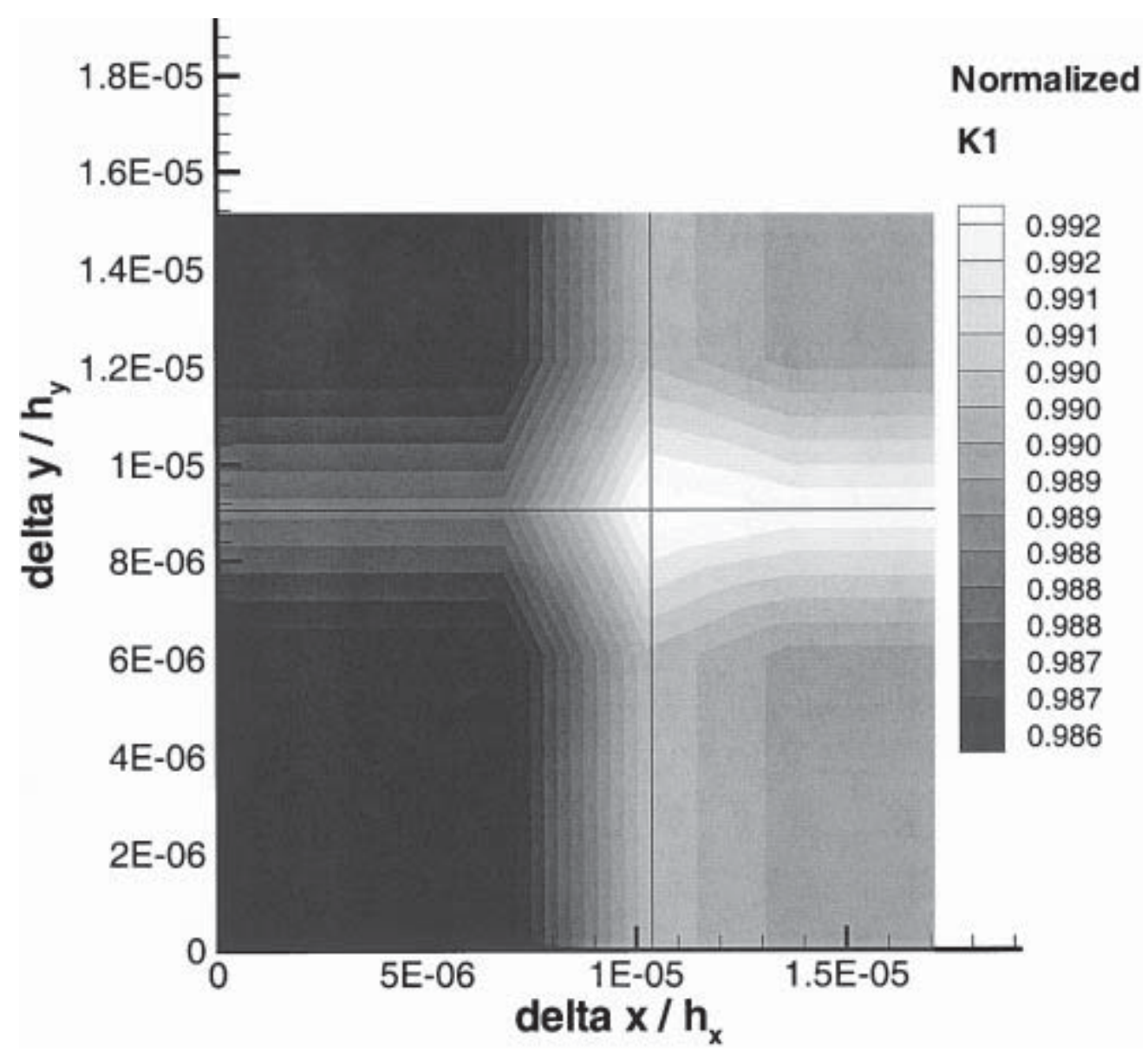

Figure 16. Robustness analysis results: normalized $K_{\mathrm{I}}$ with respect to the crack translation. The solid lines indicate element edges

The numerical results normalized by the exact solution are shown in Figure 16. The axes are normalized by the mesh spacing in the $x$-direction $\left(h_{x}\right)$, and the $y$-direction $\left(h_{y}\right)$. The accuracy of all results is excellent, and there is less than 1 per cent variation among all configurations tested.

\subsection{Shear edge crack}

In this example, we consider the edge crack geometry of Figure 14 subjected to a shear load. The plate is clamped at the bottom and a shear of $\tau=1.0 \mathrm{psi}$ is applied to the top. For this case, we examine the accuracy of the method for four different discretizations. In addition, for each discretization we consider several different domain sizes for the interaction integrals described in Section 3.4. The invariance of the calculated stress intensity factors with domain size is an indication of the quality of the numerical solution.

Four discretizations with uniform nodes and scattered nodes in an unstructured mesh are studied. The first uniform mesh consists of $13 \times 25$ nodes evenly spaced, while the second (shown in Figure 17(a)) consists of $25 \times 49$ nodes. In the table of results which follows, these meshes are denoted by struct1 and struct2, respectively. The first unstructured mesh consists of a combination of triangular and quadrilateral elements for a total of 503 nodes. The second unstructured mesh (shown in Figure 17(b)) is more refined with a total of 1955 nodes. These meshes are denoted in the table of results as unstr1 and unstr2, respectively. Figure 18 shows the unstructured mesh around the crack. 


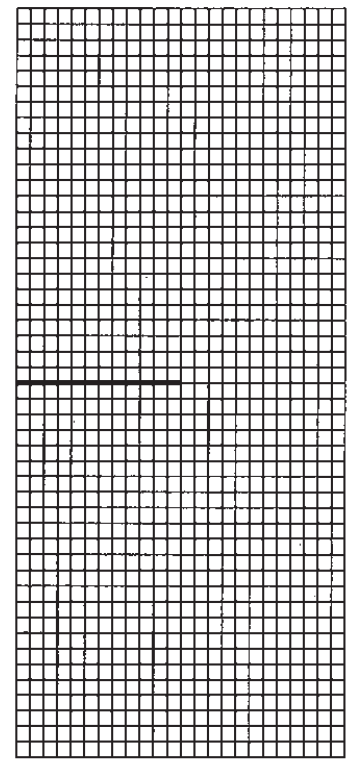

(a)

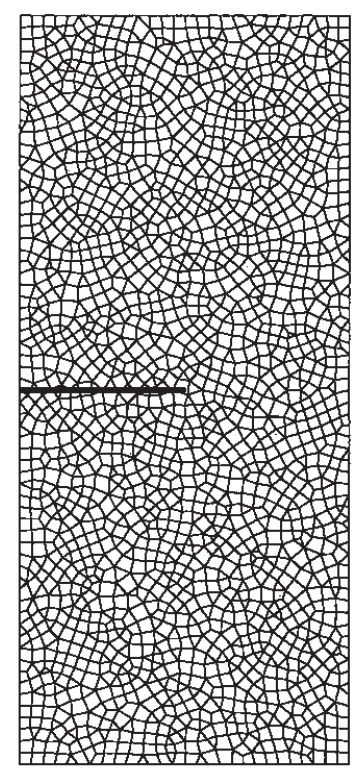

(b)

Figure 17. Meshes for edge crack: (a) structured; (b) unstructured

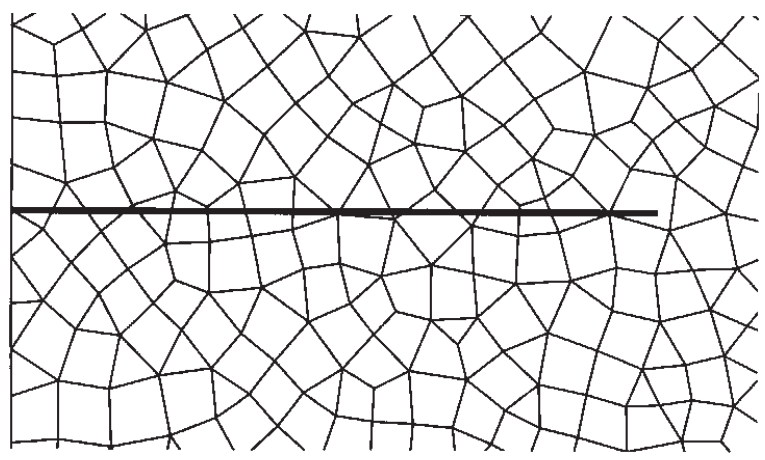

Figure 18. Unstructured mesh near the crack

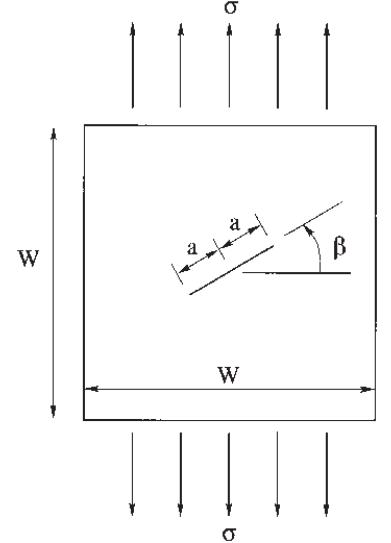

Figure 19. Plate with a centre crack at angle $\beta$

Table I. Normalized $K_{\mathrm{I}}$ values for various discretizations and domain sizes

\begin{tabular}{lllll}
\hline Mesh type & \multicolumn{2}{c}{ Structured } & \multicolumn{2}{c}{ Unstructured } \\
\hline$r_{d} / h_{\text {local }}$ & struct1 & struct2 & unstr1 & unstr2 \\
1.5 & 0.995 & 1.004 & 0.975 & 0.985 \\
2.5 & 0.986 & 0.996 & 0.961 & 0.970 \\
3.5 & 0.986 & 0.996 & 0.962 & 0.970 \\
\hline
\end{tabular}

The exact stress intensity factors for this load case are given by [13]

$$
K_{\mathrm{I}}=34.0 \mathrm{psi} \sqrt{\text { in }}, \quad K_{\mathrm{II}}=4.55 \mathrm{psi} \sqrt{\text { in }}
$$


Table II. Normalized $K_{\text {II }}$ values for various discretizations

and domain sizes

\begin{tabular}{lllll}
\hline Mesh type & \multicolumn{2}{c}{ Structured } & \multicolumn{2}{c}{ Unstructured } \\
\hline$r_{d} / h_{\text {local }}$ & struct1 & struct2 & unstr1 & unstr2 \\
1.5 & 1.016 & 1.015 & 0.932 & 1.025 \\
2.5 & 1.001 & 1.000 & 0.981 & 0.987 \\
3.5 & 1.001 & 0.9998 & 0.995 & 0.985 \\
\hline
\end{tabular}

The normalized results for the various discretizations and crack contour domains are given in Tables I and II. The results are excellent in terms of overall accuracy and domain-independence for each discretization.

\subsection{Plate with angled centre crack}

To illustrate the versatility and effectiveness of this formulation, stress intensity factors are calculated for a plate with an angled centre crack shown in Figure 19. The plate is subjected to a far field uniaxial stress as shown. In this example, $K_{\mathrm{I}}$ and $K_{\mathrm{II}}$ are obtained as a function of the crack angle $\beta$ for a uniform mesh. It is emphasized that the same mesh is used for all angles.

In this example, the plate dimensions are taken to be $W=10$ in with a half crack length of $a=0.5 \mathrm{in}$. The mesh is a uniform array of $40 \times 40$ rectangular elements. As the plate dimensions are large in comparison to the crack length, the numerical solution can be compared to the solution for an infinite plate. For the load shown, the exact stress intensity factors are given by

$$
\begin{aligned}
K_{\mathrm{I}} & =\sigma \sqrt{\pi a} \cos ^{2}(\beta) \\
K_{\mathrm{II}} & =\sigma \sqrt{\pi a} \sin (\beta) \cos (\beta)
\end{aligned}
$$

The results in Figure 20 show excellent agreement with the exact solution for the entire range of $\beta$.

\subsection{Crack growth}

As a last example, we consider the modelling of crack growth in a plate with cracks emanating from two holes subjected to a far-field tension. Figure 21(a) shows the geometry and loads under consideration. In the initial configuration, both cracks have a length of $0 \cdot 1$ in and are oriented at angles $\theta=45$ and -45 for the left and right holes, respectively.

The initial geometry is discretized with a mesh of 2650 scatterred nodes as shown in Figure 21(b). The mesh is refined towards the centre of the plate, where the cracks are expected to propagate. It is emphasized that the mesh does not conform to the crack geometries, and that the same mesh is used throughout the simulation. Figure 22 shows a closeup of the mesh near the hole.

The cracks are driven by a Paris fatigue law with the maximum circumferential stress hypothesis for the angle of crack propagation. In the initial study, the change in crack length for each iteration is taken to be a constant $\Delta a=0 \cdot 1$ in, and the cracks are grown for nine steps. The two cracks grow in a nearly symmetrical pattern, despite the fact that the mesh is not symmetric next to last configuration. Table III gives the position and stress intensity factors of the top crack tip at each stage of the simulation. In this example, the cracks eventually grow into the holes. 


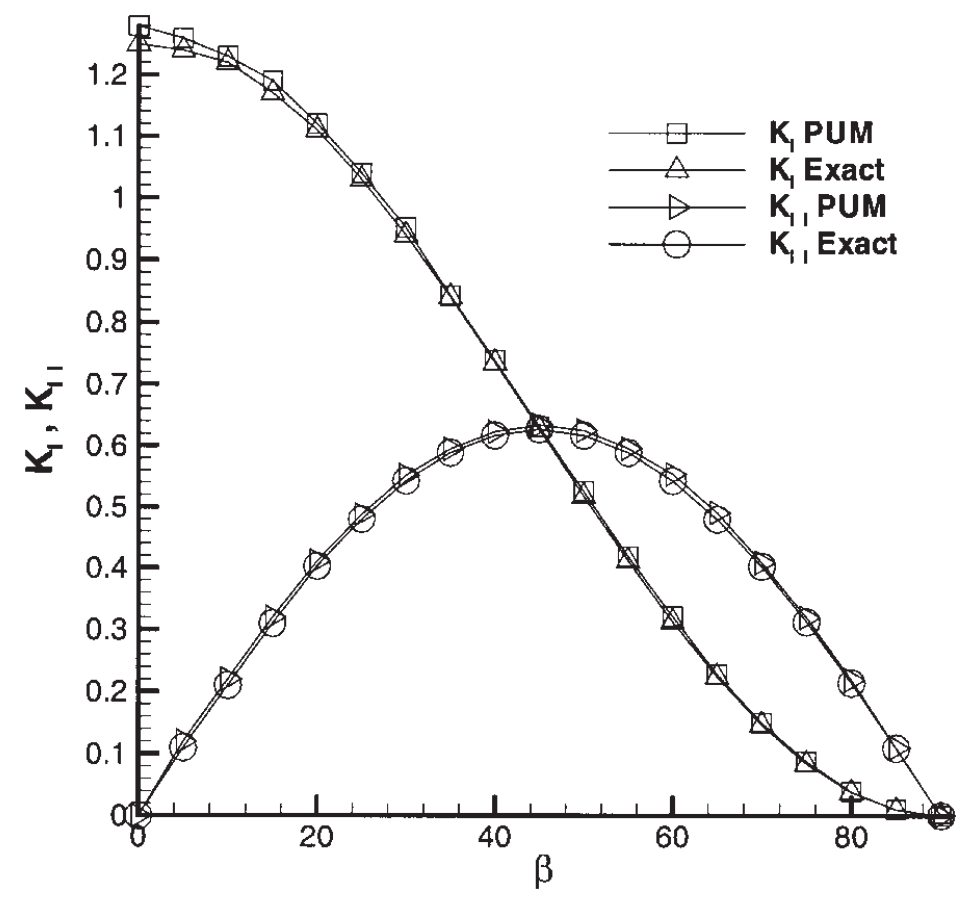

Figure 20. $K_{\mathrm{I}}$ and $K_{\mathrm{II}}$ vs. $\beta$ for a plate with an angled centre crack
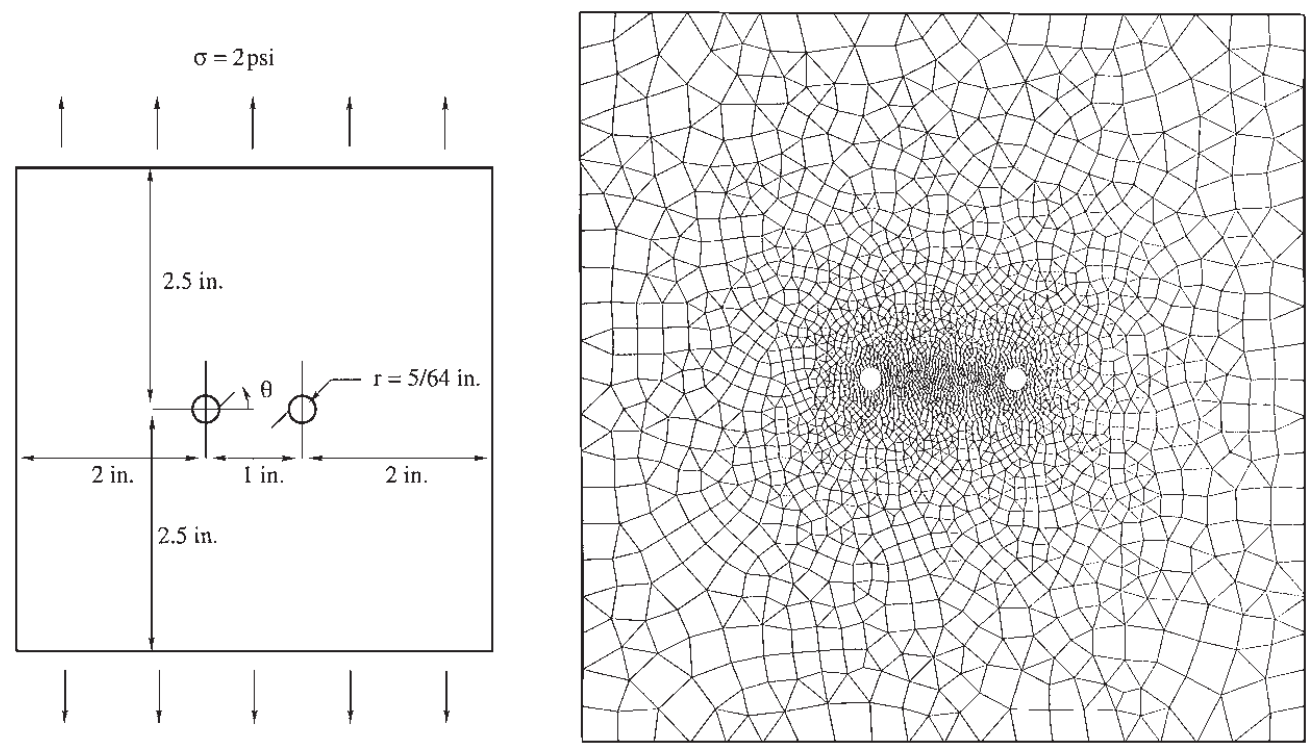

Figure 21. Description and mesh for the crack growth problem. The initial crack lengths are $0 \cdot 2$ in

We next refine the mesh in the vicinity of the crack paths, and halve the change in crack length to $\Delta a=0.05 \mathrm{in}$. The fine mesh consists of quadrilateral and triangular elements for a total of 5117 nodes. The cracks are grown for a total of 16 steps, and once again grow towards the holes. The crack tip position and stress intensity factors for the top crack tip are provided in Table IV. Figure 23 compares the final crack paths for both the coarse and fine mesh, and shows good correlation. 


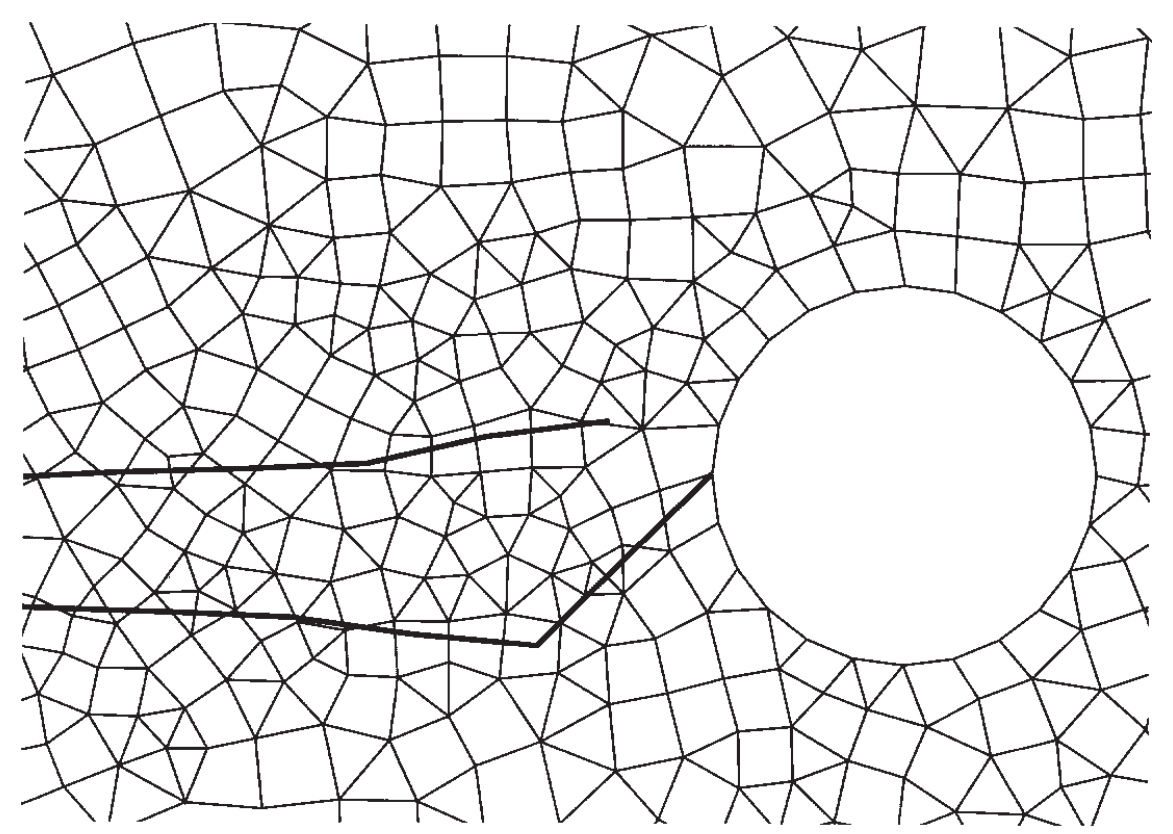

Figure 22. Mesh and final crack configuration near the right hole

Table III. Position and stress intensity factors for left crack tip. Coarse mesh results with $\Delta a=0 \cdot 1$ in

\begin{tabular}{|c|c|c|c|c|}
\hline \multirow[b]{2}{*}{ Step } & \multicolumn{2}{|c|}{ Tip position } & \multicolumn{2}{|c|}{ SIFs } \\
\hline & $x_{c}$ & $y_{c}$ & $K_{\mathrm{I}}$ & $K_{\text {II }}$ \\
\hline Initial & $2 \cdot 1488$ & 2.5707 & 0.78 & 0.65 \\
\hline 1 & $2 \cdot 2484$ & $2 \cdot 5616$ & 1.55 & -0.02 \\
\hline 2 & $2 \cdot 3482$ & $2 \cdot 5550$ & $1 \cdot 84$ & $-0 \cdot 10$ \\
\hline 3 & $2 \cdot 4481$ & $2 \cdot 5592$ & $2 \cdot 53$ & $0 \cdot 00$ \\
\hline 4 & $2 \cdot 5480$ & $2 \cdot 5634$ & $2 \cdot 31$ & 0.95 \\
\hline 5 & $2 \cdot 6312$ & $2 \cdot 5080$ & 0.92 & -0.19 \\
\hline 6 & 2.7289 & $2 \cdot 4866$ & 0.48 & -0.27 \\
\hline 7 & $2 \cdot 8153$ & $2 \cdot 5370$ & 0.56 & 0.28 \\
\hline 8 & 2.9139 & $2 \cdot 5204$ & $0 \cdot 11$ & $-0 \cdot 10$ \\
\hline
\end{tabular}

\section{SUMMARY AND CONCLUDING REMARKS}

A method has been developed for modelling crack growth by enrichment that includes the asymptotic near tip field and a Haar function. The Haar function is used away from the crack tip. Its use represents the main improvement of this technique over that presented in [3], where a mapping of the discontinuous near-tip field was employed for curved cracks. The Haar function provides a much more elegant and straightforward procedure, and is readily generalized to other problems such as those involving nonlinear materials and three dimensions. 
Table IV. Position and stress intensity factors for left crack tip. Fine mesh results with $\Delta a=0.05$ in

\begin{tabular}{lcccr}
\hline & \multicolumn{2}{c}{ Tip position } & \multicolumn{2}{c}{ SIFs } \\
\hline Step & $x_{c}$ & $y_{c}$ & $K_{\mathrm{I}}$ & \multicolumn{1}{c}{$K_{\mathrm{II}}$} \\
Initial & 2.1488 & 2.5707 & 0.79 & 0.65 \\
1 & 2.1986 & 2.5663 & 1.45 & $0 \cdot 03$ \\
2 & 2.2481 & 2.5595 & 1.57 & -0.07 \\
3 & 2.2981 & 2.5575 & 1.68 & $-0 \cdot 01$ \\
4 & 2.3481 & 2.5581 & 1.86 & $0 \cdot 01$ \\
5 & 2.3981 & 2.5562 & 2.09 & $-0 \cdot 12$ \\
6 & 2.4480 & 2.5600 & 2.56 & $0 \cdot 08$ \\
7 & 2.4980 & 2.5608 & 3.03 & 0.41 \\
8 & 2.5465 & 2.5488 & 2.64 & 0.46 \\
9 & 2.5886 & 2.5219 & 2.63 & -0.04 \\
10 & 2.6321 & 2.4972 & 2.68 & -0.28 \\
11 & 2.6820 & 2.4998 & 2.73 & 0.01 \\
12 & 2.7320 & 2.5013 & 0.57 & 0.02 \\
13 & 2.7819 & 2.5037 & 0.55 & -0.05 \\
14 & 2.8306 & 2.5151 & 0.68 & 0.03 \\
15 & 2.8802 & 2.5217 & 0.64 & -0.03 \\
\hline
\end{tabular}

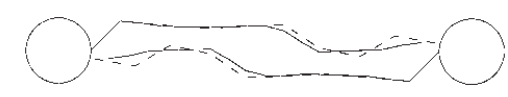

Figure 23. Crack paths for the coarse (dashed line) and fine (solid line) meshes

We were struck in the study of this technique by how accurately the stress intensity factors can be computed with relatively coarse meshes. This characteristic makes the method very promising for fatigue crack growth calculations in complex geometries, such as an engine block, where any remeshing would be prohibitively burdensome.

The method treats the crack as a completely separate geometric entity and the only interaction with the mesh occurs in the selection of the enriched nodes and the quadrature of the weak form. We have reported here quadrature schemes that use a subdivision of the elements cut by the crack, but reasonable results can be obtained with simpler schemes. The only requirement is that the quadrature scheme integrate on both sides of the discontinuity for nodes enriched with the Haar function.

In contrast to the element enrichment schemes of Benzley [16], the accuracy of this enrichment based on a partition of unity is almost independent of element size for a large range (almost any size consistent with reasonable practice). In addition, the use of crack tip elements requires transition elements and the technique tends to deteriorate as the element size near the crack tip decreases. About the only drawback of the present method is the need for a variable number of degrees of freedom per node. The partition-of-unity also has the desirable feature that the FEM equations retain the sparsity properties of the original mesh. 
The support of the Office of Naval Research and Army Research Office, to Northwestern University, is gratefully acknowledged. The authors are grateful for the support provided by the DOE Computational Science Graduate Fellowship program, to John Dolbow.

\section{REFERENCES}

1. Oliver J. Continuum modelling of strong discontinuities in solid mechanics using damage models. Computational Mechanics 1995; 17:49-61.

2. Rashid MM. The arbitrary local mesh refinement method: an alternative to remeshing for crack propagation analysis. Computer Methods in Applied Mechanics and Engineering 1998; 154:133-150.

3. Belytschko T, Black T. Elastic crack growth in finite elements with minimal remeshing. International Journal for Numerical Methods in Engineering 1999; 45(5):601-620.

4. Melenk JM, Babuška I. The partition of unity finite element method: Basic theory and applications. Computer Methods in Applied Mechanics and Engineering 1996; 39:289-314.

5. Oliver J. Modelling strong discontinuities in solid mechanics via strain softening constitutive equations. Part 2: numerical simulation. International Journal for Numerical Methods in Engineering 1996; 39:3601-3623.

6. Babuška I, Rosenzweig M. A finite element scheme for domains with corners. Numerical Mathematics 1972; 20: 1-21.

7. Grisvard P. Elliptic Problems in Nonsmooth Domains. Pitman Publishing, Inc: Boston, 1985.

8. Szabo B, Babuška I. Finite Element Analysis. Wiley-Interscience: New York, 1991.

9. Fish J. Finite element method for localization analysis. Ph.D. Thesis, Northwestern University, 1989.

10. Nuismer R. An energy release rate criterion for mixed mode fracture. International Journal of Fracture 1975; 11:245-250.

11. Erdogan F, Sih G. On the crack extension in plates under plane loading and transverse shear. Journal of Basic Engineering 1963; 85:519-527.

12. Sih G. Strain-energy-density factor applied to mixed-mode crack problems. International Journal of Fracture 1974; 10:305-321.

13. Yau J, Wang S, Corten H. A mixed-mode crack analysis of isotropic solids using conservation laws of elasticity. Journal of Applied Mechanics 1980; 47:335-341.

14. Shih C, Asaro R. Elastic-plastic analysis of cracks on bimaterial interfaces: part I-small scale yielding. Journal of Applied Mechanics 1988; 55:299-316.

15. Ewalds H, Wanhill R. Fracture Mechanics. Edward Arnold: New York; 1989.

16. Benzley S. Representation of singularities with isoparametric finite elements. International Journal for Numerical Methods in Engineering 1974; 8:537-545. 